\title{
Batteries assessing functional fitness of older people: a brief review
}

\author{
Rutkauskiene L., Lendraitiene E. \\ Department of Rehabilitation, Medical Academy, Lithuanian University of Health Sciences, Kaunas, \\ Lithuania \\ ${ }^{1}$ Corresponding author \\ E-mail: ${ }^{1}$ rutkauskienelaura@gmail.com, ${ }^{2}$ egle_77@yahoo.com
}

Received 12 November 2019; received in revised form 8 December 2019; accepted 15 December 2019 DOI https://doi.org/10.21595/chs.2019.21180

Check for updates

Copyright (C) 2019 Rutkauskiene L., et al. This is an open access article distributed under the Creative Commons Attribution License, which permits unrestricted use, distribution, and reproduction in any medium, provided the original work is properly cited.

\begin{abstract}
Regular physical activity of older people leads them to more independent life and reduces risk of illness and mortality from various chronic diseases. There are tests for muscular strength, flexibility, aerobic endurance, balance, body mass index, but for the older people is more interesting to know how they are able to do their daily life activities. Physical impairment leads to functional limitation (restriction in physical behaviors such as walking, standing up, stair climbing) which can lead to disability. The batteries contain various types of tests, ranging from basic motor skills to specific day-to-day tasks, depending on the physical activity field to be tested. The purpose of this review is to compare differences in the functional fitness assessing batteries of older adults to determine if they are common to use for physiotherapists and to help them in process of choosing.
\end{abstract}

Keywords: older people, batteries, test, functional fitness.

\section{Introduction}

Physical fitness has traditionally been associated with the more active young through to the middle-aged population. However, functional fitness is most critical for those in their senior years. It is important for older adults to have adequate strength, flexibility, and endurance to accomplish everyday tasks. Assessing these components of fitness can detect weaknesses which can be treated before causing serious functional limitations [1]. Identifying the key physiological attributes associated with functional mobility is important in developing physical assessments and in planning exercise prevention/rehabilitation programs. Based on a literature review and feedback by expert judges, the following were identified as being key physiological parameters related to functional mobility in older adults: muscular strength, aerobic endurance, flexibility, agility/dynamic balance, body mass index [2]. All physical activity assessment methods have their own advantages and disadvantages: criterion methods accurately measure physical activity, but they are expensive and unsuitable for large-scale studies; objective methods are far more likely to provide reliable assessments, but may not assess all parameters of physical activity; subjective methods are cheap and acceptable to both researchers and individuals, however they may be valid for ranking individual's behavior whereas their absolute validity to quantify physical activity is limited [3]. A key issue in clinical geriatrics and in aging studies is the assessment of physical functioning. In recent years, various physical activity batteries have been developed and used in the elderly population in which people are asked to perform several tasks and are measured using different criteria [4]. Neuropsychological Assessment Battery for memory measures and daily living tests may have clinical utility in detecting informant-rated functional impairment in dementia [5]. The new is that computerized tests minimize floor and ceiling effects by properly adjusting the level of difficulty to the cognitive abilities of the participant. These tests can also record accuracy and speed of response with a level of precision not possible in paper-and-pencil assessments. Therefore, computerized testing emerging as an attractive option compared to traditional instruments [6]. The purpose of this review is to compare differences in the functional fitness assessing batteries of older adults to determine if they are common to use for 
physiotherapists and to help them in process of choosing.

\section{Fitness assessing batteries}

Fitness in the elderly is assessed using three types of batteries:

- Health related batteries (A).

- Daily life activities assessing batteries (B).

- Comprehensive batteries (C).

Functional fitness is defined as having the physical capacity without excessive fatigue to perform normal daily activities safely and independently. As we age, we want to have the strength, endurance, flexibility, and mobility to remain active and independent so that we can take care of our own personal and household needs; do our own shopping; or participate in active social, recreational, and sport activities, if that is our choice [2]. Usually, functional fitness is tested using batteries that include a mix of health and performance monitoring, including aerobic ability measures, muscle strength and endurance, body weight and structure, flexibility, balance, and coordination [7]. The concept of health-related physical fitness was first introduced by what is known as the Toronto model on physical activity, fitness, and health (Fig. 1). According to this model, habitual physical activity can influence the physical fitness level, which can, in turn, modify the level of physical activity. The model also specifies that fitness is related to health in a reciprocal manner [8].

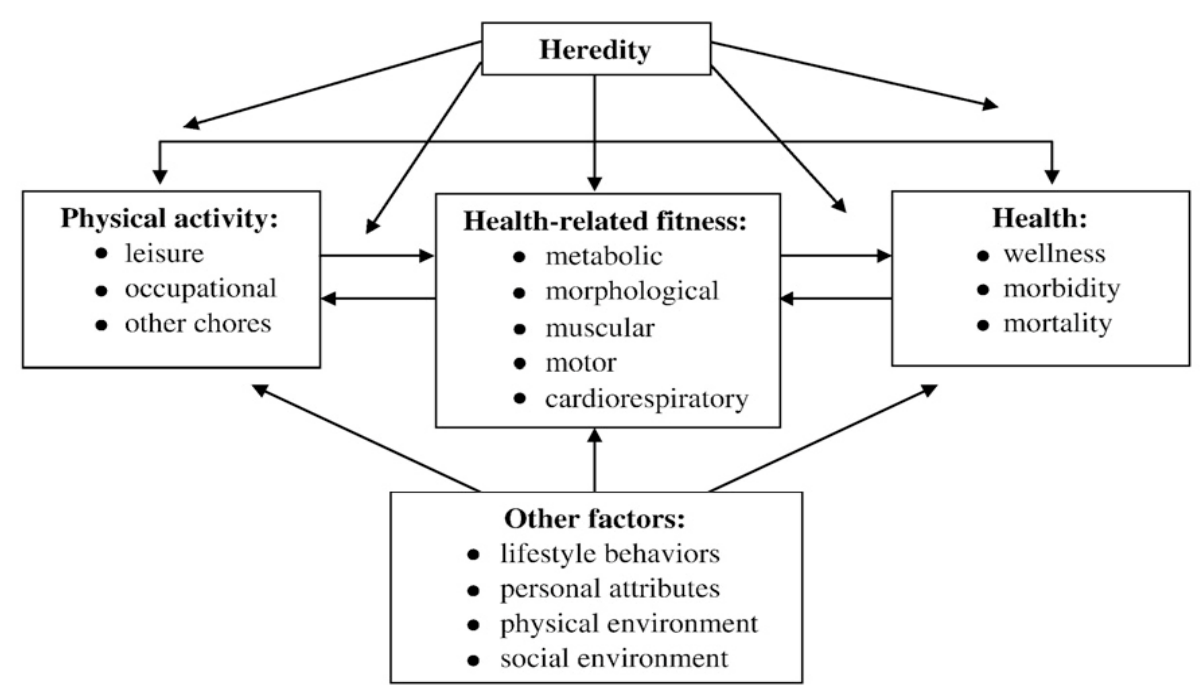

Printed with permission from Bouchard and Shepard (1994), copyright $@ 2009$ by Human Kinetics

Fig. 1. The theoretical model of the relationships among habitual physical activity, health-related fitness, and health status (Bouchard and Shephard 1994)

\subsection{American alliance for health, physical education, recreation and dance (A)}

One of the first attempts to create a particular battery for assessing the physical fitness of the elderly was carried out by the American Alliance for Health, Physical Education, Recreation and Dance (AAHPERD). The AAHPERD Physical Fitness Task Force was formed to develop an effective and appropriate health status test for older adults [9]. These are the test components: Ponderal Index - a height-weight ratio which serves as an index of body composition; Sit and Reach Test - measuring flexibility of the trunk/leg; Agility Test - testing agility/dynamic balance, rising from a chair and maneuvering around cones; Soda Pop Test - an arm and hand coordination test, in which you turn six soda pop cans over in a specified order, one at a time, as 
quickly as possible; Arm Curl Test - a seated biceps curl test which measures muscular strength/endurance of the upper body; 880 yard walk - test of aerobic endurance, walk the distance as quickly as possible.

This battery was examined several times for reliability and validity tests and once the relevant normative criteria have been identified. It has become one of the most common batteries and one of the most useful tools for databases. Some deficiencies were found: the absence of some lower body muscle function tests or the fact that some of the exercises (flexibility and aerobic endurance) can be challenging for many elderly people to perform, as well as the confirmation of a learning effect. In addition, it should be noted that the protocol should be modified in two of the exercises (coordination and strength) to improve its reproducibility. Only the leaning impact must be controlled, particularly with regard to the exercises of agility, flexibility, and coordination. The AAHPERD is a battery that can be easily managed due to its low cost and minimal requirements for space and equipment. This instrument is very useful to evaluate physical parameters associated with daily activities [4].

\subsection{Short physical performance battery (A)}

The Short Physical Performance Battery (known as SPPB or Nacional Institute on Aging battery) is an increasingly common test used to measure lower-extremity performance in older adults. SPPB was developed in the 1980s. The creation goal was to make battery for performing in any home by a single person. It includes a strength, balance, and mobility component. The SPPB involves timing performance on the following items: five chair stands (no arms), 8-ft walk test, and three hierarchical balance tests (side-by-side stance, modified tandem stance, or tandem stance) [10]. Its performance $-10-15 \mathrm{~min}$. The SPPB battery predicts mobility disability and activities of daily living disability independently, mainly through the assessment of strength, balance, and gait speed [11]. A short physical performance battery assessing lower extremity function shows correlation with self-reported impairment and mortality prediction and admission to the nursing home. Some researchers have included a level of 0 (performance level between 0 and 5) which refers to people who are unable to perform the exercises, those individuals who are unable to walk, or those situations where it is obvious that there is a risk of injury if the candidates take the test [12]. Test-retest reliability of data obtained with the SPPB is good, with intraclass correlation coefficients for measurements taken 1 week apart ranging from 0.88 to 0.92 [13]. The SPPB has been shown to be a strong predictor of decline in physical function in older adults [11].

\subsection{The Groningen fitness test for the elderly (A)}

The Groningen fitness test for the elderly (GFE) was developed as a simple method to measure components of motor fitness in groups of self-reliant adults over age 55 in field circumstances such as a sport center, gymnasium, or community center. GFE is a motor fitness evaluation tool for investigation of interrelationships between motor fitness, physical activity, health, and daily functioning [14]. This battery contains manual dexterity and reaction time checks, which are described as important physical aptitude characteristics. Those checks were combined with a questionnaire for personal wellbeing self-assessment [4]. The reliability, inter-rater, intra-rater, and internal consistency of the GFE has been demonstrated. So, it showed that GFE is a valuable measurement device for basic motor abilities such as strength, endurance, and coordination. It should be noted that the circumduction test lacks objectivity, that it has been suggested to include adequate heating before the sit and reach test, and that some prior training before the block transfer exercise would be advisable to prevent the learning impact. The endurance exercise (walking test) may not be very rigorous, considering that some people can complete it without achieving the full level of effort. GFE is used to assess the fitness level of sedentary populations and individuals affected by various diseases. Its performance for each test takes $4 \mathrm{~min}$ and the endurance test $15 \mathrm{~min}$. Its items are easy to handle and easy to carry, but requires specific equipment, so it may 
not be easy to perform in all situations [14].

\subsection{Activities of daily living (B)}

The degree to which a person can live independently largely depends on his or her ability to perform daily functional tasks known as ADLs. ADLs are the tasks defining the daily functional skills of an individual: basic (self-dressing, hygiene, etc.) and instrumental (household, shopping, etc.) [15]. ADL studies concentrate on reproducing complex, real-life activities instead of basic physiological capabilities. These measures are closer to the concept of disability (defining physical disability "as the inability to carry out independently specific roles, or activities within a given environment) than more basic skills testing [16].

\subsection{Performance oriented mobility assessment (B)}

The Tinetti Performance Oriented Mobility Assessment (POMA) is one of the earliest and most widely used batteries designed to assess balance, gait, and fall risk in older adults. It includes an evaluation of balance under perturbed conditions (such as while rising from a chair, after a nudge, with eyes closed, and while turning), as well as an evaluation of gait characteristics (including gait initiation, step height, length, continuity and symmetry, trunk sway, and path deviation). A score less than 19 out of 28 has a sensitivity of $68 \%$ and a specificity of $88 \%$ for predicting an individual who will have two or more falls [17]. The test takes about 10 to 15 minutes to administer, and the interrater reliability of this test is good.

\subsection{Senior fitness test (B)}

Rikli and Jones developed the Senior Fitness Test (SFT) to assess the functional fitness of older adults aged over 60 years. The SFT is a set of six tests that are economical, comprehensive, and easy to administer and include measures of lower-body strength (30-second chair stand), upper-body strength (30 second arm curl), aerobic endurance (2-minute step-in-place), lower-body flexibility (sit-and-reach), upper-body flexibility (back scratch), and agility/ dynamic balance (8-foot up-and-go). Rikli and Jones (1999a) developed the SFT to evaluate and monitor the physical fitness of older adults with the goal of identifying and treating limitations in functional fitness. As defined by Rikli and Jones (2001), "functional fitness is having the physical capacity to perform normal everyday activities safely and independently without undue fatigue" [16]. The Senior Fitness Test is tested for reliability, with an intra-class correlation coefficient (ICC) ranging from 0.8 to 0.98 on the different items. Validity of the different items was tested when there were "gold standards" [18].

\subsection{The functional independence measure $(C)$}

The FIM instrument was developed as part of the Uniform Data System for Medical Rehabilitation at the U.S. - shows the extent of the condition of the patient and how much nursing he needs. Periodic reassessment will monitor patient quality changes over time while presenting data to determine the outcomes of rehabilitation. The task force created a seven-level scale for performance measurement and selected items that assessed self-care, sphincter control, transfers, locomotion, communication, and social cognition. Each level marks major behavioral gradation from dependence to independence. This scale rates patients performance of an activity based on their need for assistance from another person. FIM shows the time and effort that another person must expend to serve the dependent needs of the disabled patient so that the patient can achieve and maintain a certain quality of life. The FIM tool is a disability aid, not an impairment. This battery does not have to show what the patient thought to be able to do or might be able to do under different conditions. In the initial development stages, the FIM instrument was found to have high face and content validity as judged by both clinicians and a panel of experts. The FIM 
instrument was highly correlated with other measures of disability in the patient groups studied, thus indicating that the FIM instrument has good concurrent validity with other measures of disability. The FIM instrument was highly reliable across the rating clinicians. The total FIM rating intraclass correlation coefficients is 0.96 [19].

Very important aspect for authors was how to administer batteries easy and score and have minimal requirements with respect to equipment, time, space, and training. Looking to these aspects we made table and we also added batteries creation dates and duration to perform them (Table 1). It is always important for physiotherapists to know these aspects after choosing testing batteries.

Table 1. The years of creation, time for performance, easily administered of fitness assessing batteries

\begin{tabular}{|c|c|c|c|}
\hline Fitness assessing batteries & Year of creation & Time in minutes & Easily administered \\
\hline AAHPERD & 1990 & 20 & + \\
\hline SPPB & 1980 & 10 & + \\
\hline GFE & 1990 & $30-40$ & - \\
\hline ADL & 1950 & individual & - \\
\hline POMA & 1986 & $10-15$ & + \\
\hline SFT & 1999 & $30-40$ & + \\
\hline FIM & 1987 & $15-20$ & - \\
\hline
\end{tabular}

\section{Conclusions}

Most functional measures have limitations. First a patient's performance is examined under a limited set of environmental conditions; thus, it may not always predict actual performance in more complex environments. In addition, most functional tests provide little insight into the quality of movement used to accomplish the task and provide no way to identify specific neuronal or musculoskeletal subsystems within the body responsible for a decline in performance.

For many authors it was important that test items be reliable (intra-class correlation coefficient for all previously mentioned batteries includes 0,80-0,99 limits) and valid; be sensitive enough to detect expected changes in performance due to aging or to exercise intervention; be able to assess a wide range of performance levels, from borderline frail to highly fit; be easy to administer and score and have minimal requirements with respect to equipment, time, space, and training; be socially acceptable and motivating to older people.

Batteries assessing functional fitness of older people on all previous lists can be easily performed by physiotherapists. Even some tests can be done by home conditions.

\section{References}

[1] Wood R. Testing the Elderly. Topend Sports Website, 2008, https://www.topendsports.com/testing/elderly.htm.

[2] Rikli R. E., Jones C. J. Senior Fitness Test Manual. USA Human Kinetics, 2001, p. 2-23.

[3] Janonienė R., Sobutienė A., Valintėlienė R. Physical activity assessment methods. Visuomenès Sveikata, Vol. 3, Issue 66, 2014, p. 10-22, (in Lithuanian).

[4] Varela S., Ayan C., Cancela J. M. Batteries assessing health related fitness in the elderly: a brief review. European Review of Aging and Physical Activity, Vol. 5, 2008, p. 97-105.

[5] Ashendorf L., Alosco M. L., Bing Canar H., et al. Clinical utility of select neuropsychological assessment battery tests in predicting functional abilities in dementia. Archives of Clinical Neuropsychology, Vol. 33, Issue 5, 2018, p. 530-540.

[6] Brenkel M., Shulman K., Hazan E., Herrmann N., Owen A. M. Assessing capacity in the elderly: comparing the MoCA with a novel computerized battery of executive function. Dementia and Geriatric Cognitive Disorders Extra, Vol. 7, 2017, p. 249-256.

[7] Capranaica L., Tiberi M., Figura F., Osness W. Comparison between American and Italian older adult performances on the AAHPERD functional fitness test battery. Journal of Aging and Physical Activity, Vol. 9, 2001, p. 11-19. 
[8] Bouchard C., Shephard R. J. Physical activity, fitness, and health: the model and key concepts. Physical Activity, Fitness, and Health: International Proceedings and Consensus Statement, 1994, p. 77-88.

[9] Clark B. Test for fitness in older adults. AAHPERD Fitness Task Force. Journal of Physical Education, Recreation and Dance, Vol. 60, Issue 3, 1989, p. 66-71.

[10] Bergland A., Strand B. H. Norwegian reference values for the short physical performance battery (SPPB): the Tromsǿ study. BMC Geriatrics, Vol. 19, 2019, p. 216.

[11] Guralnik J., Simonsick E., Ferruci L., Glynn R., Berkman L., Blazer G., Scherr P., Wallace R. A short physical performance battery assessing lower extremity function: association with selfreported disability and prediction of mortality and nursing home admission. The Journals of Gerontology, Vol. 49, 1994, p. 85-94.

[12] Ostir G. V., Ottenbacher K. J., Fried L. P., Guralnik J. M. The effect of depressive symptoms on the association between functional status and social participation. Social Indicators Research, Vol. 80, 2007, p. 379-392.

[13] Ostir G. V., Volpato S., Fried L. P., et al. Reliability and sensitivity to change assessed for a summary measure of lower body function: results from the women's health and aging study. Journal of Clinical Epidemiology, Vol. 55, 2002, p. 916-921.

[14] Lemmink K., Han K., De Greef M., Rispens P., Stevens M. Reliability of the Groningen fitness test for the elderly. Journal of Aging and Physical Activity, Vol. 9, 2001, p. 194-212.

[15] Dobek J., White K., Gunter K. The effect of a novel ADL based training program on performance of activities of daily living and physical fitness. Journal of Aging and Physical Activity, Vol. 15, 2006, p. 13-25.

[16] Wilkin L. D., Haddock B. L. Functional fitness of older adults. Activities, Adaptation and Aging, Vol. 35, Issue 3, 2011, p. 197-209.

[17] Goldberg A., Alexander N. B. Performance Oriented Mobility Assessment (POMA). Fifth Edition, Primary Care Geriatrics/Gait and Mobility, 2007.

[18] Langhammer B., Stanghelle J. K. Functional fitness in elderly Norwegians measured with the senior fitness test. Advances in Physiotherapy, Vol. 13, 2011, p. 137-144.

[19] Gupta A. Measurements Scales Used in Elderly Care. CRC Press, 2008. 